\title{
Research on Physical Damage Characteristics of Table Tennis Players and the Precautionary Measures
}

\author{
Ming Lu \\ Physical Education College, Baicheng Normal University, Baicheng, 137000, China
}

Keywords: Physical damage characteristics, Table tennis players, Precautionary measures

\begin{abstract}
There is no physical contact between table tennis players, so it is not easy to hurt as basketball, football, free combat and other items. Even if injured, the injury is rarely too severe to completely participate in the competition, which cannot get enough attention. This paper analyzes the characteristics of physical injury of table tennis players, including the characteristic of injured regions and the reasons for injuries, and gives some protection suggestions to reduce sports injuries.
\end{abstract}

\section{Introduction}

Table tennis is the development of our country and achieved the best sports, whether in sports or basic level mass level, table tennis has made impressive achievements in the development of our country, known as China's "national sport" table tennis sports interest because of its high entertainment value. And, at all levels of the school have been vigorously promote and carry out. How to guarantee the high quality of table tennis training is particularly important, all things are possible sports injuries and physical damage, unscientific tennis exercise methods not only for students' physical development and school table tennis atmosphere promoting effect, it will cause the students physical damage and inefficiency of table tennis sports teaching school. To cause damage of table tennis and solutions, we should start with the movement characteristics of table tennis starting: table tennis is a ball for the change of the campaign; it changes the ball, block, high on the movement of the motor skills requirements. Table tennis requires a good comprehensive ability of sports, that is, athletes are required to have good endurance, body coordination and response agility. Athletes need according to the ball line, in a very short time, rapid response and make action to hit the ball, and the ball movement requires coordination of all parts of the body, to use joint sensitivity to complete. These characteristics of table tennis make the body consume a lot of physical energy and have a great impact on the joints. So, on the long table tennis players, if there is no scientific and reasonable exercise plan, did not bring damage to the prevention program of table tennis, may be on the movement of various parts of body joints, Achilles tendon and muscle injury. Based on the principle of table tennis technical movements, this paper summarizes the vulnerable parts of table tennis and puts forward reasonable prevention measures.

\section{Physical Damage Characteristics of Table Tennis Players}

Characteristic of Injured Regions. The wrist is mainly composed of bone shapes, in view of the anatomical characteristics, we can make all kinds of wrist movements are so flexible, it is because small much, so it is easy to cause the main cause of wrist injury. The smaller range of activities of the wrist, when a ball is hit, the entire arm to speed leverage, make quick pronation abduction movements of wrist, the wrist is not good if the flexibility or strength of wrist joint is poor prone to sports injuries. Most of the injuries in table tennis were lumbosacral. In table tennis, the body should always keep the upper body leaning forward, then, the lumbar spine ligament always keep the upper body leaning forward also long time contraction tension, many athletes do not pay attention to relax at the end of the exercise, resulting in excessive local fatigue and exhaustion that loss. In table tennis, almost every 
shot, all cannot do without the waist movement, waist to muscle, if insufficient preparation, large muscle groups have not yet overcome inertia, is also a major cause of waist injury in sports. In the course of playing, the knee joints are always in a semi flexion position, and the ligaments around the joints are in tension and traction. If there is no adaptive activity in the knee before the exercise, it is easy to cause the injury of the medial and lateral ligaments of the knee joint when it is suddenly over stretched. In addition, the knee injury and athletes play, pull type and choppers play, the activity of the lower limbs range, large amplitude and speed, causing the knee joint burden, so easily lead to injury. Therefore, to adjust the playing style according to personal circumstances, can also strengthen the knee joint exercise to reduce the damage, such as by standing, squat to strengthen the muscle strength of femoral head.

Injury Characteristic of Different Models. In the pitch under the same injury rate was significantly higher than that of hand technique and tactics in the game, shot under the same chance to play the curveball injured is significantly greater than the linear ball. Chop at the Dongguan University of Technology public sports classroom training among the least likely to hurt. Exercise pen hold curveball and grip pull pull arc ball students are mainly caused by the shoulder and waist injury, fast ball and grip pen hold exercise arc arc fast ball is subject to more students mainly shoulder and knee injuries, the main body, the damage caused by the fast ball pen for knee and wrist, and chop technique will cause the waist injury. Comprehensive analysis: the curve ball will cause shoulder joint injury, and fast break ball will cause knee joint injury. All play the most likely cause of damage to the body is straight pull arc ball, because this play is the main use of single pull loop of forehand, basically is to rely on burst points, waist muscle force is the key to achieve a curve ball, the waist force fully, pulled out of the loop impact force is large and stable, fully burst. The sudden rapid start and sudden braking of the shoulder joint is the key to achieve the accuracy of hitting the ball. The sudden start and braking of the shoulder joint is likely to result in tension and fatigue of the shoulder muscles, resulting in a strain. In this way, the shoulder and wrist is the most vulnerable, the slightest mistake will cause different degrees of damage. Most likely to lower extremity injuries play is chopping play. Because of the use of the chopping athletes play the lower limb activity is frequent and large strength, so often in the semi flexion knee in an unstable state in a relatively long time, easy to be injured; at the same time, in order to maximize the return of space and time, athletes should use the waist turning, if the athlete's waist strength is insufficient or flexibility is not strong, it is very likely to cause injury. The use of arc type athletes play the most vulnerable part of the waist and shoulders. The reason is that the athletes want to pull out of the high quality of the loop, it must have strong waist strength, especially the side grip. If the right hand to get the player on the right side of the waist requires muscle strength, muscle is left to a certain extent relative relaxation, left hand shot athletes. If the opposite is the case in the course of play, to coordinate the muscle position and strength, it is very easy to cause lumbar sprain. While in the playground loop when is a standard action arm drive arm, this is the heaviest burden of shoulder joint, so easy to cause the shoulder joint injury. At the same time, when using the pen hold grip pulls the arc ball, with great racket hand on the side of the waist muscles, while the other side muscles are more relaxed, on both sides of the waist muscle tension force is not the same, will form a poor muscle strength, it is easy to cause the lumbar muscle sprain.

Reasons for Injuries. Most people think that table tennis is a net separation movement; there is no direct physical contact and confrontation, so the probability of injury will be very low, very safe. Because of this inherent idea, general table tennis training students cannot form enough attention, training and competition are not ready for the first half of the campaign, some students even ignore the preparatory activities, began to high strength and high load of table tennis training and competition, this is a very a reasonable method of table tennis. Before formal training and competition, the body function of the human body is generally in a lower level. To make the body quickly into the excited state, it is necessary to warm up before exercise. The purpose of warming up exercise is to regulate the movement state of the body and prevent the occurrence and aggravation of the injuries. Reasonable and effective preparation exercise is based on the characteristics of table tennis events and the combination of the actual physical performance of athletes. If at the beginning 
of table tennis, the amount of preparatory activities being too large, it will backfire, is the body of the excitement in the formal training and competition at the beginning has been in the past, the body has been reduced to a state of exhaustion, the body cannot make the body more inert, excited again, aggravated the probability of injury; if for lack of exercise, the amount is too small, so, when the athlete's body function at the start of formal training and competition, is unable to achieve the best. Without adequate body function regulation, the joints and muscles of the human body cannot be activated, so that the intensity of the training and competition, but also easy to cause physical damage. Therefore, reasonable preparation should follow the movement characteristics of table tennis sports, physical function combined with the movement itself to the design, according to the difference of movement function of the body to formulate a scientific and feasible, the preparation plan, step by step, be ready to move on.

\section{Precautionary Measures of Physical Damages of Table Tennis Players}

Strengthen Self-protection Awareness Education. Attention should be paid to the full preparation before exercise and the active relaxation after exercise. Full preparations of science can make the muscles of the human body, organs, nervous system gradually into motion, reduce the viscosity of human muscle, muscle stretch, and restore the reflex action, make full preparations for the formal training or competition. Sports psychology has great effect on sports injury of athletes, if excessive tension in the table tennis training and competition of loose, even angry and so on, he didn't notice the changes in your body function, such as some parts of the pain cannot first time to react, to take protective measures, thus, will greatly increase the risk of bodily injury. Preparations for the general requirements of athletes was fever, sweating slightly as well, not excessive movement of the official function level is not in the best condition, and according to the old wound and easily hurt parts strength and tensile proper practice, for the prevention of sports injury and disease occurrence has obvious effect, weaken the physiological role of cohesion time at the same time, attention and prevention between formal sports activities have ready. The active relaxation after exercise can relieve fatigue after exercise, and accelerate the migration of metabolites produced during exercise, which can effectively reduce the injury caused by the absence of muscle recovery during the second movement. The general relaxation part mainly includes jogging and stretching relaxing activities. We should strengthen the understanding of sports injuries and diseases, and respect the doctor's advice and arrangement. In the usual exercise and training, we should pay more attention to the prevention of sports injuries and diseases, and earnestly implement the policy. If there are sports injuries and diseases, we should respect the doctor's advice and arrangement, attach importance to the rehabilitation training, avoid the emergence of chronic strain and re injury.

Command Correct Technical Movement. Table tennis is a very sophisticated technical sports program. From the beginning of the grip, the athletes should be in the correct guidance of coaches, correct learning table tennis skills and footwork, to master the basic skills of table tennis, and should follow the principle of gradual, not be anxious, it has a very important role in the prevention of sports injury. Table tennis is a kind of test technology of sports, so the learning is to carefully listen to the professional guidance of teachers, especially in the early stage of the standard action more to develop good skills, because if not early grasp of the scientific skills, so after the training is hard to correct come, and scientific manipulation is an important aspect of health protection of athletes. In table tennis, the use of technical movements is correct, directly related to whether athletes can win the game, but also related to whether the muscle damage. Table tennis is a type of technical movement, skill requirements are very high, beginners must be in the correct guidance of coaches, grasp the reasonable technical movement, and then through long-term repeated practice, the formation of automatic action. Many beginners didn't have the skill, right to crash, did not understand the importance of technical movements, thus forming subconscious misconceptions, blind practice, it will form a long-term effect of sports performance sports injury.

Arrange Proper Exercise Intensity. In the teaching of table tennis, athletes should follow the principle of gradual progress, coaches should be arranged to make large, medium and small 
combination, followed by periodic features. Practice should shorten the exercise time, should increase the intensity, delay the practice time should reduce the intensity of the load. To avoid the complex technical movement exercises and excessive exertion under the condition of body fatigue and partial overload, and to deal with the relationship between training time and training intensity. A large part of sports injury is caused by excessive exercise load, so the scientific arrangement of exercise has become an important task for each coach. Scientific and reasonable arrangement of exercise load for effective physical potential and improve athletic performance has a positive effect, but excessive training sometimes scores increased rapidly, and even counterproductive. Although excessive training in a short period of time, the results will be significantly improved, but for the long term, this training is not appropriate, often is an important reason for the occurrence of sports injuries. The characteristics of table tennis sports determine the particularity of table tennis training. Table tennis is a unilateral muscle activity, especially the waist and shoulder the most obvious because of the long-term load strength training, hand side limb movement than the other side of the thick, waist muscles will be more nervous than the contralateral muscle. Proper strengthening of the old wounds and vulnerable parts of the strength and flexibility of quality exercise, for common sports injuries have good preventive effect. At the same time table tennis difficult technical action also requires athletes have the corresponding physical quality, it plays an important role in the rational use of table tennis technique and tactics in the game. Therefore, in peacetime should pay attention to the balanced development of sports, fitness training to enhance awareness of limb strength training, pay attention to the development of opposing muscle strength, promote the harmonious development of the body, especially the waist if not pay attention to the long-term, there may be a scoliosis and other sports injury.

\section{Conclusion}

Table tennis is a popular and favorite sport in the world. In China, table tennis is also known as China's national ball. So the scientific and reasonable of the table tennis research, through the reasonable scheme to help athletes reduce the damage burden in training, but this is not responsible for the health of athletes, more is to contribute to the society.

\section{References}

[1] Fu Haiyan, Jia Junjie, Qin Hao, et al. Analysis Of The Characteristics Of Injury In Elite Table Tennis Players In Henan Province [J]. Jouranl of Anhui Sports Science, 2015, 36(3): 51-54.

[2] Ding Lingling. The Investigation and Analysis on the Injury of Young Ping Pang Players [J]. Journal of Nanjing Sport Institute (Natural Science), 2015, 14(1): 73-76.

[3] Shang Xuedong, Wang Zongbao, Li Wei. The effects of individualized exercise training on rotator cuff injury in elite table tennis players [J]. Chinese Journal of Sports Medicine, 2015, 37(2): 112-115.

[4] Li li, Jin linzi. Table Tennis Athletic In uries and Preventive Measures [J]. Journal of Henan Normal University (Natural Science Edition), 2012, 40(2): 178-180. 